Tom Farrell, Shuja Reagu, Suruchi Mohan*, Riham Elmidany, Feras Qaddoura, Ebtehag Elfadil Ahmed, Gillian Corbett, Stephen Lindow, Salwa Mohammed Abuyaqoub and Majid Ali Alabdulla

\title{
The impact of the COVID-19 pandemic on the perinatal mental health of women
}

https://doi.org/10.1515/jpm-2020-0415

Received August 28, 2020; accepted September 17, 2020;

published online September 25, 2020

\section{Abstract}

Objectives: The physical health impact of the coronavirus disease infection (COVID-19) has received attention worldwide; however, data around the psychological impact of the pandemic is still emerging and little has been reported on psychological effects among vulnerable groups. This study was undertaken with the aim of studying the impact of the COVID-19 pandemic and related restrictions on perinatal mental health among women in Qatar.

Methods: A cross- sectional survey of women accessing maternity services in Qatar was carried out during the months of June and July 2020 at the local peak of the pandemic. Background data including relevant demographic details, pregnancy and mental health history, concerns, as well as helpful stress-reducing factors reported by women was collected. Depression and anxiety symptomatology was studied using the Patient Health Questionnaire Anxiety-Depression Scale (PHQ-ADS).

Results: The survey results revealed a high prevalence of anxiety and Depressive symptomatology (34.4 and 39.2\% respectively), based on PHQ-ADS scoring. These rates appeared much higher than the reported pre-pandemic prevalence and were not affected by occupation, previous mental health problems or pregnancy complications.

Tom Farrell and Shuja Reagu are first co-authors of the work.

*Corresponding author: Suruchi Mohan, Department of Obstetrics, Sidra Medicine, Doha, Qatar, E-mail: suruchimohan@yahoo.com Tom Farrell, Riham Elmidany, Feras Qaddoura, Ebtehag Elfadil Ahmed and Salwa Mohammed Abuyaqoub, Women's Wellness and Research Center, Hamad Medical Corporation, Doha, Qatar

Shuja Reagu and Majid Ali Alabdulla, Department of Psychiatry, Hamad Medical Corporation, Doha, Qatar

Gillian Corbett and Stephen Lindow, Coombe Women and Infants University Hospital, Dublin, Ireland

2 Open Access. (C) 2020 Tom Farrell et al., published by De Gruyter. (cc) BY License.
Women's most commonly reported concerns as well as coping factors are discussed.

Conclusions: Results indicate a marked increase in anxiety and depressive symptoms during the COVID-19 pandemic, among pregnant and puerperal individuals, who constitute a vulnerable group with respect to mental health morbidity. These findings can be used to inform public health interventions, among which, consideration should be given to routine mental health screening of vulnerable groups during major health crises.

Keywords: anxiety; COVID-19; depression; mental health; pandemic; perinatal; pregnancy; stress.

\section{Introduction}

From its start as an outbreak of pneumonia in China in December, 2019 [1], the spread of Coronavirus-2 infection has evolved dramatically into a major worldwide health crisis. The World Health Organization declared Coronavirus disease (COVID-19) a pandemic on March 11, 2020 [2].

As the pandemic unfolded, public concern about the risks to life and health, inadequate healthcare services and economic consequences grew. As part of infectioncontainment strategies, governments around the world imposed unprecedented restrictions, and these resulted in the compromise of personal and social liberty. Within a month of the declaration of the pandemic, $90 \%$ of the world's population was subject to some restriction of movement to limit infection spread [3]. The impact of the ensuing social isolation and loneliness along with the worries about risks of the infection and its economic fallout would appear likely to have an effect on mental health of the population. Indeed, increased mental health morbidity including anxiety and depression, in a similar context, has been reported previously with fears arising from the SARS outbreak [4]. Epidemiological data on the adverse impact of COVID-19 on mental health is emerging $[5,6]$. The preliminary data has also shown that women have demonstrated a more significant psychological impact from the pandemic [6]. 
Pregnancy can make women particularly vulnerable in relation to mental health morbidity and it has been reported that pregnant and puerperal women show higher rates of anxiety and depressive symptomatology compared to non-pregnant women of child-bearing age [7]. Given the added effects of the pandemic situation, this group may be particularly at risk of mental health effects. Depression and anxiety in pregnancy is associated with serious adverse effects for mother and baby, including risks of miscarriage, premature birth, low birth weight, lower Apgar scores, more cesareans for maternal request and higher postnatal depression $[7,8]$. Therefore, there is a need to explore the psychological impact of the COVID-19 pandemic within this vulnerable group as well as investigate successful coping strategies employed by pregnant women so that these may be used to inform public health strategy and identify foci for future interventions.

This survey of pregnant and puerperal women in Qatar was conducted with the aim of exploring rates of anxiety and depression symptomatology during the COVID-19 pandemic, comparing these to pre-pandemic data and identifying successful supportive strategies.

\section{Materials and methods}

Formal ethics approval for this study was obtained from the organizational Ethics Committee of Hamad Medical Corporation, Qatar where this study was undertaken. In line with the committee's guidance, an information sheet detailing the project aims and scope was given to participants and informed consent for participation obtained.

The state tertiary care maternity hospital in Qatar, where this study was conducted, is the largest provider of maternity services and has a delivery rate of approximately 18,000 deliveries per year (unpublished data). Based on this and the known prevalence for anxiety and depression amongst pregnant women in Qatar, OpenEpi [9] was used to calculate the required sample size of 285 for $95 \%$ confidence intervals.

A cross-sectional survey design was agreed and a questionnaire designed for data collection. The survey was available to the participants both English and Arabic. The survey questionnaire comprised two parts where the first section had questions aimed at collecting relevant demographic data, information about current pregnancy, past mental health history, self-reported concerns in relation to the pandemic and coping factors perceived useful. The second part of the questionnaire was the Patient Health Questionnaire AnxietyDepression Scale (PHQ-ADS) [10] - which combines the PHQ-9, GAD-7 scales, both of which have been previously extensively used, validated separately [10] and for which a validated Arabic translation is available [11]. A score of five and above is indicative of anxiety on the GAD-7 scale and of depression on the PHQ-9 scale. Incremental score level cut offs are used as indicators of the severity of anxiety and depressive symptomatology [12].

The survey was conducted in the months of June and July, 2020, when the COVID-19 pandemic in Qatar had peaked [13]. Participation was offered to all pregnant and puerperal women attending the hospital at the antenatal clinics, obstetric emergency unit and the inpatient maternity unit. Exclusion criteria included inability to consent or to engage with the written survey format due to literacy or learning difficulty issues or severe physical morbidity. The survey was closed when 288 responses were obtained.

Data collected were transferred to SPSS version 23.0 (Armonk, NY: IBM Corp) for analysis. Descriptive statistics were performed and non-parametric analyses of continuous variables were cross referenced with nationality, occupation, education, pregnancy complications and previous mental health problems.

\section{Results}

A total of 288 women completed the questionnaire. The general characteristics of the participant group are outlined in Table 1. The Qatari women were younger and had more children than the women from the Philippines, India and other counties. The mean age and parity was 28.6 years and 2.1 children compared to 33.7 years and 1.2 children for the Philippines and 30.7 years and 0.8 children for the Indian women and 30.5 and 1.9 children for the other group (analysis of variance, $\mathrm{F}=6.5$ and 6.7 respectively, $\mathrm{p}<0.0001)$. There was no difference in the gestational age between the nationalities. Overall 170 (59\%) of women were not employed, 147 (51.7\%) were university educated and 170 (59\%) had no pregnancy problems.

Women were primarily concerned about their pregnancy (143 women) and their family and children (189 women). Only 9 (3.1\%) of the women had previous mental health problems with $7 / 9$ being anxiety and depression.

The major sources of help were information from staff (118), or televised media (138) but few used exercise to help cope with stress (9).

Using the GAD-7 and the PHQ-9 scores of five or greater; the overall prevalence of anxiety and depression in the group was calculated at $34.4 \%$ and $39.2 \%$ respectively.

The results of the GAD-7 score and the PHQ-9 are outlined in Figure 1. There was a positive correlation between the individual scores (Pearson correlation $=0.814$, $\mathrm{p}<0.01)$.

Table 2 details the GAD-7 and PHQ-9 scores indicative of anxiety and depression severity among the participants. Within the group whose scores were indicative of anxiety (at least 5), nearly half of the group (46.6\%) scored in the moderate to severe range (10-21). Whereas among those with depression scores (5 or more), $45.1 \%$ scored in the moderate to severe range (10-27).

Non-parametric analysis between anxiety (GAD-7) and depression (PHQ-9) scores and occupation (Kruskal-Wallis 3.04, $\mathrm{p}=\mathrm{NS}$ and 3.56, $\mathrm{p}=\mathrm{NS}$ respectively) and previous 
Table 1: General background, concerns and coping strategies of the 288 patients studied.

\begin{tabular}{|c|c|}
\hline & 288 respondents \\
\hline Mean (SD) Age (Y) & $30.5(5.3)$ \\
\hline Mean (SD) Gestation (Weeks) & $26.1(14.3)$ \\
\hline Mean (SD) Number children & $1.7(1.7)$ \\
\hline \multicolumn{2}{|l|}{ Country of origin } \\
\hline Qatari origin & $53(18.4 \%)$ \\
\hline Philippines origin & $33(11.5 \%)$ \\
\hline Indian origin & $42(14.6 \%)$ \\
\hline Other origin & $160(55.6 \%)$ \\
\hline \multicolumn{2}{|l|}{ Education } \\
\hline School education & $59(20.9 \%)$ \\
\hline College education & $62(21.5 \%)$ \\
\hline University education & $149(51.7 \%)$ \\
\hline None & $16(5.6 \%)$ \\
\hline \multicolumn{2}{|l|}{ Occupation } \\
\hline Unemployed & $170(59 \%)$ \\
\hline Healthcare & $48(16.7 \%)$ \\
\hline Hospitality & $3(1.0 \%)$ \\
\hline Administration & $21(7.3 \%)$ \\
\hline Other & $45(15.6 \%)$ \\
\hline \multicolumn{2}{|l|}{ a Pregnancy problems } \\
\hline Diabetes & 73 \\
\hline Hypertension & 17 \\
\hline Other & 29 \\
\hline None & $170(59 \%)$ \\
\hline${ }^{\mathrm{b}}$ Previous mental health problems & $9(3.1 \%)$ \\
\hline \multicolumn{2}{|l|}{${ }^{\mathrm{a}}{ }^{\mathrm{b}}$ Worries } \\
\hline Pregnancy & 143 \\
\hline Finances / job & 27 \\
\hline Children / family & 189 \\
\hline Other & 15 \\
\hline \multicolumn{2}{|l|}{${ }^{a}$ Sources of help } \\
\hline Information from staff & 118 \\
\hline TV & 138 \\
\hline Family/friends & 47 \\
\hline Hobbies & 23 \\
\hline Exercise & 9 \\
\hline Other & 12 \\
\hline
\end{tabular}

${ }^{\mathrm{a}}$ Some women completed more than one item and up to five items.

${ }^{\mathrm{b}} 1$ case missing.

mental health problems ( $\mathrm{n}=287$. Mann-Whitney $\mathrm{U}-0.98$, $\mathrm{p}=\mathrm{NS}$ and -1.68 ) were not significant.

Nationality was not associated with high GAD-7 scores (Kruskal-Wallis 4.58, NS) but PHQ-9 scores were significantly lower in Philippine than Qatar women (Kruskal-Wallis 3.04, $\mathrm{p}<0.014$ corrected for multiple tests). Education was significantly associated with the GAD-7 and PHQ-9 with college educated women demonstrating significantly lower scores than university educated women (Kruskal-Wallis -3.58, $\mathrm{p}<0.002$ and -3.74 , $\mathrm{p}<0.001$ respectively, corrected for multiple tests).

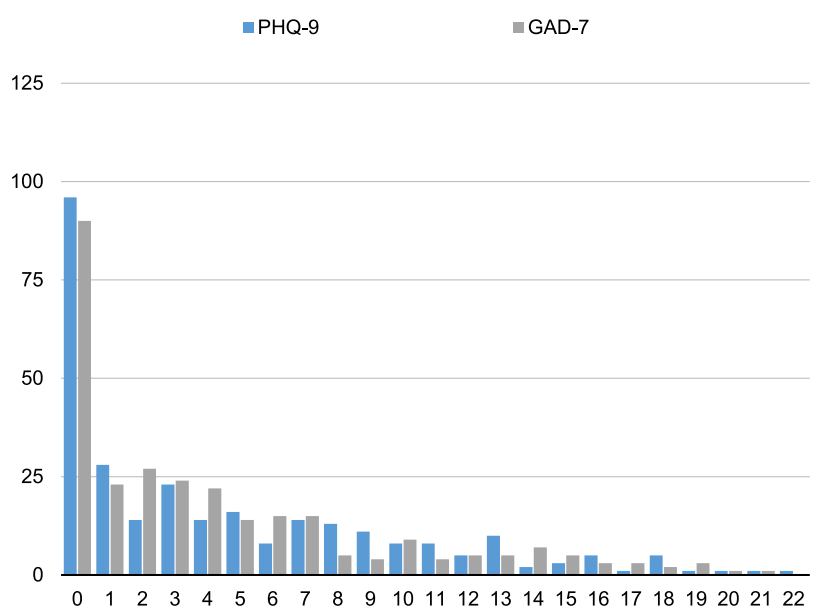

Figure 1: The responses of 287 women to the GAD-7 anxiety score and 288 women to the PHQ-9 depression score.

Pearson correlation between the individual scores $=0.814, p<0.01$.

Women with any pregnancy complications did not score higher on either score than those without (MannWhitney $\mathrm{U} 0.22$ and -0.35 , both $\mathrm{p}=\mathrm{NS}$ ).

The respondents were reclassified by gestational age into first and second trimester $(\mathrm{n}=54)$, third trimester $(n=184)$ and postnatal $(n=50)$. There was a significant difference in the PHQ-9 score between the three groups (Kruskal Wallis 19.51, $\mathrm{p}<0.0001$ ) with postnatal scores being significantly higher than either of the two antenatal groups. The GAD -7 score was also significantly different (Kruskal Wallis 26.08, $\mathrm{p}<0.0001 . \mathrm{n}=183$ for the third trimester group) and postnatal scores were significantly higher than either of the antenatal groups. There was no significant difference in PHQ-9 or GAD-7 scores between the third trimester and first/second trimester antenatal groups.

In summary, more than a third of women scored at least five on both the anxiety (34.4\%) and depression (39.2\%) ratings, despite a very low proportion of the group having self-reported preexisting mental health problems. Individual anxiety and depression scores were positively correlated. The main concerns women expressed were for others rather than themselves. Information from healthcare workers and televised pandemic related information was cited most commonly as helpful; and use of exercise to relieve stress was low.

\section{Discussion}

To our knowledge, this is the first study form the Middle Eastern and North African (MENA) region that explores the psychological impact of COVID 19 in the pregnant and 
Table 2: GAD-7 and PHQ-9 scores indicative of anxiety and depression.

\begin{tabular}{lr}
\hline GAD-7 Score & Number of participants \\
\hline $5-9$ (Mild anxiety) & 53 \\
$10-14$ (Moderate anxiety) & 30 \\
$15-21$ (Severe anxiety) & 16 \\
Total & 99 \\
PHQ-9 score & \\
$5-9$ (Mild depression) & 62 \\
$10-14$ (Moderate depression) & 33 \\
$15-19$ (Moderately severe depression) & 15 \\
$20-27$ (Severe depression) & 3 \\
Total & 113 \\
\hline
\end{tabular}

puerperal population. It is also the first study to utilize the Patient Health Questionnaire Anxiety-Depression Scale (PHQ-ADS) to study the perinatal health impact of the pandemic. The components of PHQ-ADS have arguably the greatest evidence for validity based on their extensive use. These tools have been utilized in numerous studies of depression and anxiety symptoms and have been adopted across the globe by variety of healthcare settings. These are available free to access in over 80 languages [10, 12]. The main finding of the study was a high prevalence of anxiety (34.4\%) and depressive (39.2\%) symptomatology among the women studied, where only $3.1 \%$ of the group had reported pre-existing mental health conditions. Only a few studies have explored anxiety and depression in pregnant and puerperal women in the context of the COVID-19 pandemic and our rates appear to be comparable to these. A multicenter cross-sectional study from China reported a $34.2 \%$ prevalence of depressive symptoms following the confirmation of human to human transmission in China in January 2020 [14]. A Canadian study on anxiety and depressive symptoms, in 1987 pregnant individuals in relation to the pandemic, reported depressive symptoms in $37 \%$ and anxiety symptoms in $56.6 \%$ of the participants [15]. A third study was conducted on 260 pregnant women in Turkey and reported a prevalence of depressive symptomatology of $35.4 \%$ [16]. These findings are concerning when the reported prevalence rates of depression and anxiety in the pregnant population before the pandemic are considered. A systematic review published before the pandemic reported a global prevalence of perinatal depression and anxiety as 12 and 22\% [17]. However, these globally reported rates cannot be accurately extrapolated to Qatar with its distinct demographic composition (high expatriate population) as well as unique culture. Comparing our findings to local pre-pandemic rates, in Qatar, the prevalence of depression in the general population is $13.8 \%$, that of anxiety disorders is $12.1 \%$, and rates are higher at 22 and $21.4 \%$ for the female population [18]. A recent smaller study on 128 women from Qatar put the prevalence of depression among pregnant women at $27.3 \%$ [19].

In addition, analysis did not reveal any association of anxiety and depression scores with occupation, pregnancy complications, gestational age or previous mental health illness; therefore, given that rates of these mental health conditions have increased substantially over the pandemic period, this could be attributed to the psychological impact of the pandemic and related restrictions.

The main concerns that this study group identified in relation to the Coronavirus infection, were of worries for the pregnancy and worries for their families. Research on pregnant women from Italy, has shown that the key concerns women had were centered around the health of others during the pandemic [20]. This has been echoed by present results. Additionally, the findings of this study showed significantly higher rates of anxiety and depression symptomatology in postnatal respondents when compared to pregnant participants. This is in contrast to previously reported higher rates of anxiety and depression in the antenatal period compared to the postnatal phase $[21,22]$. This could potentially be a manifestation of women's concerns for family members discussed above with the new addition to their families.

In relation to coping strategies, women in Qatar most valued information about the pandemic and precautions to take while pregnant, and their most valued sources of this help were maternity staff and televised public health information. This finding provides an insight that could inform bespoke public health education initiatives in the current and future health crisis. This also reiterates the important role healthcare staff plays in alleviating anxiety and stress in such situations. In this respect, professionals should be supported within healthcare service delivery and utilized by the organization to enable them to do justice to this important role.

There appeared to be very little reliance on physical activity as a stress-relieving mechanism. Exercise has been previously shown to reduce anxiety and depressive symptoms on pregnant women [23] and therefore could be an effective strategy to enhance mental well-bring. A study on pregnant women during the COVID-19 pandemic, revealed an association between higher physical activity and decreased anxiety and depression symptoms [15]. Cultural beliefs in this region do not encourage exercise in pregnancy and it is traditionally believed exercise in pregnancy poses risks; and as such levels of physical activity during pregnancy are low in the Middle East region 
with $18 \%$ of pregnant women being sedentary and $51.4 \%$ only engaging on light physical activity [24]. This traditional belief could be a potentially contributory factor to low uptake of exercise within this group. This area merits further exploration and could form one focus of health promotion and education activities to encourage the use of this evidence- based strategy to promote mental well-being and reduce psychological morbidity.

\section{Limitations}

While this study sheds some preliminary light on the impact of COVID-19 on the mental health of pregnant women, it is limited by the fact that it relies on self-reported data. This is particularly relevant when, locally, mental health problems still carry a social stigma [25] which may have further influenced self-reporting of symptoms by individuals in this cultural context.

Secondly, the COVID-19 pandemic is a new, rapidly evolving problem that may have a short life or may have multiple recurrences. This study was an analysis at a single time point and the true picture of mental health may show a deteriorating or a compensatory picture in the medium term.

\section{Conclusions}

The results of this study show a clinically significant problem with anxiety and depressive symptomatology during the COVID-19 pandemic among pregnant and postnatal women who constitute a vulnerable group from the mental health perspective. This increase appears to be attributable to pandemic related concerns and stresses arising from associated social restrictions, and the main concerns of these women are related to the safety of the unborn child and that of family members. These results should prompt considering the implementation of screening for mental health morbidity among pregnant women at times of major health crises. Women reported that access to information was helpful. The uptake of physical exercise is low. These finding can help inform public health initiatives and guide targeted health education campaigns in this region.

Research funding: None declared.

Author contributions: All authors have accepted responsibility for the entire content of this manuscript and approved its submission.
Competing interests: Authors state no conflict of interest. Informed consent: Informed consent was obtained from all individuals included in this study.

Ethical approval: Formal ethics approval for this study was obtained from the organizational Ethics Committee of Hamad Medical Corporation, Qatar where this study was undertaken.

\section{References}

1. Wang C, Horby PW, Hayden FG, Gao GF. A novel coronavirus outbreak of global health concern. The Lancet 2020;395:470-3.

2. Cucinotta D, Vanelli M. WHO declares COVID-19 a pandemic. Acta Biomed: Atenei Parmensis 2020;91:157-60.

3. Connor P. More than nine-in-ten people worldwide live in countries with travel restrictions amid COVID-19 [Internet]. Pew Research Center 2020. Available from: https://www. pewresearch.org/fact-tank/2020/04/01/more-than-nine-in-tenpeople-worldwide-live-in-countries-with-travel-restrictionsamid-covid-19/ [Accessed 27 Aug 2020].

4. Maunder R, Hunter J, Vincent L, Bennett J, Peladeau N, Leszcz M, et al. The immediate psychological and occupational impact of the 2003 SARS outbreak in a teaching hospital. CMAJ (Can Med Assoc J) 2003;168:1245-51.

5. Pfefferbaum B, North CS. Mental health and the Covid-19 pandemic. N Engl J Med 2020;383:510-12.

6. Wang C, Pan R, Wan X, Tan Y, Xu L, Ho CS, et al. Immediate psychological responses and associated factors during the initial stage of the 2019 coronavirus disease (COVID-19) epidemic among the general population in China. Int J Environ Res Publ Health 2020;17:1729.

7. Bennett HA, Einarson A, Taddio A, Koren G, Einarson TR. Prevalence of depression during pregnancy: systematic review. Obstet Gynecol 2004;103:698-709.

8. Grigoriadis S, Graves L, Peer M, Mamisashvili L, Tomlinson G, Vigod SN, et al. Maternal anxiety during pregnancy and the association with adverse perinatal outcomes: systematic review and meta-analysis. J Clin Psychiatr 2018;79. https://doi.org/10. 4088/jcp.17r12011.

9. Dean AG, Sullivan KM, Soe MM. OpenEpi: open source epidemiologic statistics for public health, version. www. OpenEpi.com, updated 2013/04/06. Available from: https:// www.openepi.com/SampleSize/SSPropor.htm [Accessed 22 May 2020].

10. Kroenke K, Spitzer RL, Williams JB, Löwe B. The patient health questionnaire somatic, anxiety, and depressive symptom scales: a systematic review. Gen Hosp Psychiatr 2010;32: 345-59.

11. Sawaya H, Atoui M, Hamadeh A, Zeinoun P, Nahas Z. Adaptation and initial validation of the patient health questionnaire-9 (PHQ9) and the generalized anxiety disorder-7 questionnaire (GAD-7) in an Arabic speaking Lebanese psychiatric outpatient sample. Psychiatr Res 2016;239:245-52.

12. Manea L, Gilbody S, McMillan D. Optimal cut-off score for diagnosing depression with the Patient Health Questionnaire (PHQ-9): a meta-analysis. CMAJ (Can Med Assoc J) 2012;184: E191-6. 
13. World health Organisation. WHO Coronavirus Disease (COVID-19) Dashboard [Internet]. Covid19.who.int. WHO;. 2020. Available from: https://covid19.who.int/region/emro/country/qa [Accessed 27 Aug 2020].

14. Wu Y, Zhang C, Liu H, Duan C, Li C, Fan J, et al. Perinatal depressive and anxiety symptoms of pregnant women during the coronavirus disease 2019 outbreak in China. Am J Obstet Gynecol 2020;223: 240-e1.

15. Lebel C, MacKinnon A, Bagshawe M, Tomfohr-Madsen L, Giesbrecht $\mathrm{G}$. Elevated depression and anxiety symptoms among pregnant individuals during the COVID-19 pandemic. J Affect Disord 2020;277:5-13.

16. Durankuş F, Aksu E. Effects of the COVID-19 pandemic on anxiety and depressive symptoms in pregnant women: a preliminary study. J Matern Fetal Neonatal Med 2020:1-7. https://doi.org/10. 1080/14767058.2020.1763946.

17. Woody CA, Ferrari AJ, Siskind DJ, Whiteford HA, Harris MG. A systematic review and meta-regression of the prevalence and incidence of perinatal depression. J Affect Disord 2017;219:86-92.

18. Bener A, Abou-Saleh MT, Dafeeah EE, Bhugra D. The prevalence and burden of psychiatric disorders in primary health care visits in Qatar: too little time?. J Fam Med Prim Care 2015;4:89.

19. Naja S, Al-Kubaisi N, Chehab M, Al-Dahshan A, Abuhashem N, Bougmiza I. Psychometric properties of the Arabic version of
EPDS and BDI-II as a screening tool for antenatal depression: evidence from Qatar. BMJ open 2019;9: e030365.

20. Ravaldi C, Wilson A, Ricca V, Homer C, Vannacci A. Pregnant women voice their concerns and birth expectations during the COVID-19 pandemic in Italy. Women Birth [Internet]; 2020. https://doi.org/10.1016/j.wombi.2020.07.002.

21. Dennis CL, Falah-Hassani K, Shiri R. Prevalence of antenatal and postnatal anxiety: systematic review and meta-analysis. $\mathrm{Br}$ J Psychiatr 2017;210:315-23.

22. Underwood L, Waldie K, D'Souza S, Peterson ER, Morton S. A review of longitudinal studies on antenatal and postnatal depression. Arch Wom Ment Health 2016;19: 711-20.

23. Demissie Z, Siega-Riz AM, Evenson KR, Herring AH, Dole N, Gaynes BN. Physical activity and depressive symptoms among pregnant women: the PIN3 study. Arch Wom Ment Health 2011;14: 145-57.

24. Papazian T, El Osta N, Hout H, Chammas DE, El Helou N, Younes H, et al. Pregnancy physical activity questionnaire (PPAQ): translation and cross cultural adaption of an Arabic version. PloS One 2020;15: e0230420.

25. Zolezzi M, Alamri M, Shaar S, Rainkie D. Stigma associated with mental illness and its treatment in the Arab culture: a systematic review. Int J Soc Psychiatr 2018;64:597-609. 